\title{
Powder Microelectrodes for Capillary Electrophoresis with Amperometric Detection
}

\author{
Shen Hu, Min Zhang, Dai-Wen Pang, ${ }^{\dagger}$ and Jie-Ke Cheng \\ Department of Chemistry, Wuhan University, Wuhan 430072, P. R. China
}

\begin{abstract}
In this paper, we first report the use of powder microelectrodes (PMEs) as the amperometric detector for thiols, hydrazine and hydroxylamine in capillary electrophoresis (CE). This PME, with graphite-supported metalloporphyrin powder as catalyst, offers a few advantages as an amperometric detector in CE. Concentration limit of detection and mass limit of detection obtained for L-cysteine at the PME could reach $88 \mathrm{nM}$ and 230 amol, respectively. The response of thiols at the PME was very reproducible since the electrode fouling was reduced. A relative standard deviation of peak heights $(n=8)$ of $1.8 \%$ and a relative standard deviation of migration time $(n=8)$ of $1.0 \%$ can be obtained for L-cysteine. The material packed in the PME can easily be changed. Hence, it is very convenient to choose suitable catalysts to detect special analytes, and this specially designed microelectrode should be applicable to many other compounds of biological interest.
\end{abstract}

(Received December 28, 1999; Accepted April 21, 2000)

Since its introduction a decade ago, capillary electrophoresis (CE) with amperometric detection has become a powerful analytical tool. ${ }^{1,2}$ High sensitivity combined with high efficiency and ultrasmall injection volume has made this technique a useful method for the analysis of microenvironments such as single cells. ${ }^{3}$ In early studies about this technique, carbon fiber microelectrodes were often employed for the direct amperometric detection of electroactive compounds such as phenols and catecholamines. With the development of this method, some other types of microelectrodes have been employed to extend its application area. For example, Au microelectrodes have been used for pulsed amperometric detection of carbohydrates ${ }^{4}$ or metal ions, ${ }^{5}$ copper microelectrodes for amperometric detection of amino acids and peptides, ${ }^{6,7}$ and mercury-film microelectrodes for metal ions $^{8}$ and thiols. ${ }^{9}$ In addition, chemically modified microelectrodes have also been used in CE to improve the detection sensitivity. Cobalt phthalocyanine-modified electrode was used to determine thiols, ${ }^{10}$ and platinum particle-modified microelectrodes to determine hydrazines. ${ }^{11}$

PME is a potentially useful technique for electroanalytical chemistry since it can increase the detection sensitivity significantly. ${ }^{12,13}$ In this paper, we demonstrate the first application of PME in CE-amperometry. The PME was prepared by chemically etching the tip of a $\mathrm{Pt}$ microdisk electrode to form a microcavity, then packing it with graphitesupported cobalt porphyrin powder. This specially designed electrode shows high sensitivity and good reproducibility for the detection of thiols. It was also applied to detect hydrazine, hydroxylamine and 2-mercapto ethanol in CE.

\section{Experimental}

\section{Apparatus}

The CE system with end-column amperometric detection was

$\doteqdot$ To whom correspondence should be addressed. constructed in our laboratory. Electrophoresis was carried out in fused-silica capillaries with an i.d. of $25 \mu \mathrm{m}$ and an o.d. of $375 \mu \mathrm{m}$ (Yongnian Optical Fiber Factory, Hebei, China). The capillaries were washed with $0.1 \mathrm{M} \mathrm{NaOH}$, double-distilled, deionized water and operating electrolyte prior to use. A highvoltage dc power supply ( $30 \mathrm{kV}$, Huazhong University of Science and Technology, Wuhan, China) was employed to apply the electrophoretic separation voltage. Platinum wires were used as the electrodes for applying high voltage.

The end-column amperometric detector employed was similar to that described previously. ${ }^{14}$ Detection was performed using a two-electrode configuration with an SCE reference electrode. The specific working electrode used here was a PME. The position between the capillary and the working electrode, mounted on an XYZ-micromanipulator (Wuhan Instrument Corp., Wuhan, China), was measured with an optical scale under a microscope from both top view and side view directions. The working electrode could not be placed in contact with the detection end of the capillary, since it would make the powder of the PME come loose and would increase the noise of the detector. Hence, the electrode was placed directly in front of the capillary at a distance of $\sim 2 \mu \mathrm{m}$. The working potential was controlled by an HPD-1 potentiostat (Yanbian Electrochemical Instrument Factory, Jilin, China), and the electrochemical currents produced at the detector were measured by a picoammeter (Department of Chemistry, Wuhan University, Wuhan, China) and recorded by a chart recorder (Sichuan Instrument Corp., Sichuan, China). The detector together with the electrochemical cell was housed in a Faraday cage to reduce noise. Upon installation of a new PME in the CE-amperometric detection system, no special pretreatment procedures or other operations were performed, other than applying the desired working potential and waiting roughly 20 min for the background current to stabilize. Typically, the same PME, once installed, could be used for a period of 1 month without removal from the CE system.

Cyclic voltammetric experiments were conducted using a Model 901-pA electrochemical microanalyzer (Department of 


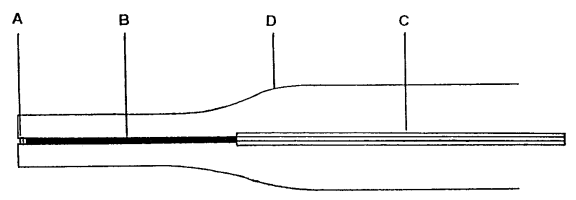

Fig. 1 Schematic diagram of PME. A, powder; B, Pt wire; C, Ag wire; D, glass tube.

Chemistry, Wuhan University, Wuhan, China) connected to an $X Y$-recorder (Dahua Instrument Corp., Shanghai, China). A two-electrode configuration was employed, since the results were identical with those obtained using a three-electrode configuration. The electrochemical cell was housed in a Faraday cage to reduce noise.

\section{Microelectrode construction}

The platinum microdisk electrode was prepared by sealing a length of platinum wire ( $30 \mu \mathrm{m}$-diameter), connected to a silver lead, in a 1-mm-i.d. capillary tube. The tip containing the sealed platinum wire was polished with abrasive paper (Liuyu Abrasive Material Corp., Jiangsu, China) and sonicated in acetone and double-distilled and deionized water.

PME was constructed from the platinum microdisk electrode. The platinum wire at the tip of the platinum microdisk electrode was etched in aqua regia for about $20 \mathrm{~min}$ so that a microcavity of $c a .20 \mu \mathrm{m}$ deep was formed. After the microcavity was washed and dried, it was packed with graphite-supported cobalt porphyrin powder (or graphite powder only for blank PME) by grinding the etched tip of the platinum microelectrode on the surface of flat glass, with a PME obtained. The cobalt porphyrin is cobalt tetrakis(4-trimethoxyphenyl)porphyrin supported on graphite and treated at $600^{\circ} \mathrm{C}$ in nitrogen atmosphere for $4 \mathrm{~h}$. A schematic diagram of a PME is shown in Fig. 1. The construction of PME is very simple and the powder packed in the PME can be easily changed by sonication, so it is very convenient to choose suitable catalysts to detect special analytes. For a refabrication of the microelectrode, we just need to take off the powder from the microcavity by ultrasonication and then pack it again. Each time the microcavity was packed by grinding on the powder till no more powder could be stuffed into the microcavity. Since the volume of the microcavity is constant, the amount of the powder packed each time could be almost the same. So the current response is quite similar.

Construction of the carbon fiber microelectrode (CFME) has been described before. ${ }^{15}$

\section{Reagents}

Unless stated otherwise, all the chemicals were of analyticalreagent grade. L-Cysteine (CYS) and reduced glutathione (GSH) were obtained from Shanghai Biochemistry Reagents Corporation (Shanghai, China). 2-N (morphorino) ethane sulfonic acid (MES) was purchased from Fluka (Buchs, Switzerland). 2-Mercapto ethanol was obtained from Life Technologies (Grand Island, NY, USA). The operating electrolytes for separation and detection were $25 \mathrm{mM}$ MES and $20 \mathrm{mM}$ phosphate buffer, and the $\mathrm{pH}$ values were adjusted with $\mathrm{NaOH}$. Stock solutions of analytes were prepared daily with buffer solutions, stored at $4^{\circ} \mathrm{C}$, and diluted to desired concentrations before use. All solutions were prepared with double-distilled and deionized water, and were filtered through a $0.22-\mu \mathrm{m}$ cellulose acetate filter (Shanghai Institute of Medical Industry).

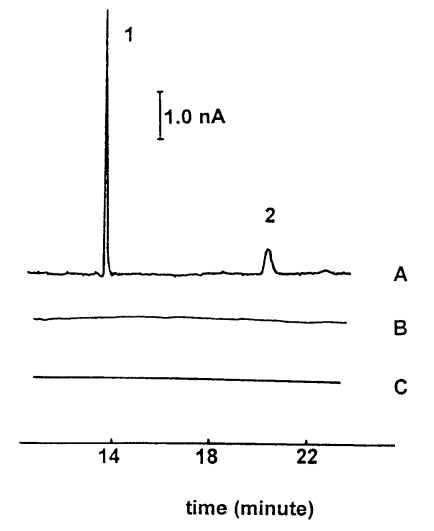

Fig. 2 Electropherograms of $500 \mu \mathrm{M}$ CYS (1) and $500 \mu \mathrm{M}$ GSH (2) at CoP/PME (A), platinum microdisk electrode (B) and CFME (C). Capillary, $25 \mu \mathrm{m} \times 70 \mathrm{~cm}$; buffer, $25 \mathrm{mM}$ MES (pH 6.0); operating voltage, $15 \mathrm{kV}$; injection, $6 \mathrm{~s}$ at $15 \mathrm{kV}$; detection potential, $1.0 \mathrm{~V}$ for PME and 1.2 V for CFME and platinum microelectrode (vs. SCE).

\section{Results and Discussion}

\section{Graphite-supported cobalt porphyrin/PME (CoP/PME)}

The electrocatalysis of cobalt porphyrin toward the oxidation and reduction of some small molecules has been investigated in our previous studies. ${ }^{16-18}$ In the present case, a pair of redox peaks at $\sim+0.47 \mathrm{~V}$ appeared with the CoP/PME in background electrolyte, while no peak was observed with a blank PME, suggesting that the peaks result from the oxidation and reduction of the porphyrin in the PME. The redox peaks are very steady, indicating no loss of the graphite-supported cobalt porphyrin powder. In addition, greatly reduced overpotential and enhanced oxidation current were observed for the oxidation of CYS at PME, indicating that the PME has catalytic activity toward the electro-oxidation of thiols such as CYS, which coincides with our previous results. ${ }^{16,18}$ One possible mechanism for the electro-oxidation of thiols (RSH) at the CoP/PME is as follows: ${ }^{18}$

$$
\begin{aligned}
& \mathrm{RSH} \stackrel{K_{1}}{\rightleftharpoons} \mathrm{RS}^{-}+\mathrm{H}^{+} \\
& \mathrm{LCo}^{\mathrm{II}} \mathrm{Y}^{+} \mathrm{RS}^{-} \stackrel{K_{2}}{\rightleftharpoons} \mathrm{LCo}^{\mathrm{II}}\left(\mathrm{RS}^{-}\right)+\mathrm{Y} \\
& \mathrm{LCo}^{\mathrm{II}}\left(\mathrm{RS}^{-}\right) \longrightarrow \mathrm{LCo}^{\mathrm{II}}(\mathrm{RS} \cdot)+\mathrm{e}^{-} \text {(rate-determining step) } \\
& 2 \mathrm{LCo}^{\mathrm{II}}\left(\mathrm{RS}^{-}\right) \longrightarrow 2 \mathrm{LCo}^{\mathrm{II}}+\mathrm{RSSR}^{\longrightarrow} \\
& \mathrm{LCo}^{\mathrm{II}}+\mathrm{RS}^{-} \longrightarrow \mathrm{LCo}^{\mathrm{II}}\left(\mathrm{RS}^{-}\right)
\end{aligned}
$$

Here, $\mathrm{LCo}^{\mathrm{II}}$ represents the catalyst coordinated on the powder and Y stands for the axial ligand.

\section{CE-amperometric detection using CoP/PME}

CFME and platinum microelectrodes are most popularly used in electroanalysis. These two types of microelectrodes provide high $S / N$ ratios, resulting in high detection sensitivity. To evaluate the performance of the system using CoP/PME, we first compared the results and those obtained at CFME and platinum microelectrodes. Figure 2 shows the capillary electropherograms of equimolar CYS, GSH using a PME, platinum microdisk electrode and CFME, respectively. MES was chosen as the electrophoretic electrolyte because the high resistance of its buffer provides low detector noise. The 


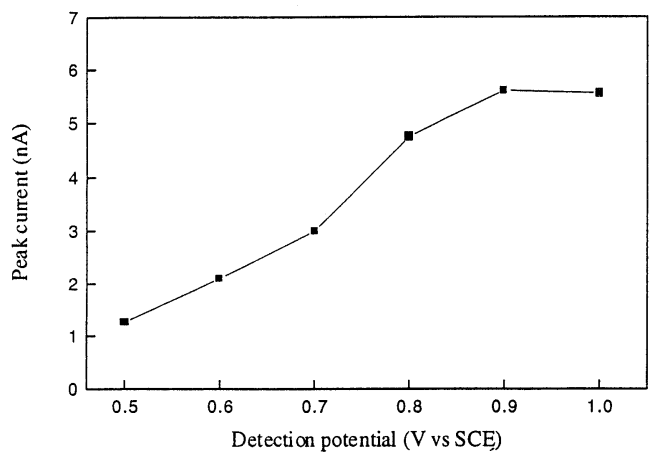

Fig. 3 Hydrodynamic voltammogram of CYS at CoP/PME. Capillary, $25 \mu \mathrm{m} \times 70 \mathrm{~cm}$; buffer, $25 \mathrm{mM}$ MES (pH 6.0); operating voltage, $15 \mathrm{kV}$; injection, $6 \mathrm{~s}$ at $15 \mathrm{kV}$; detection potential, $1.0 \mathrm{~V}(v s$. SCE); CYS concentration: $500 \mu \mathrm{M}$.

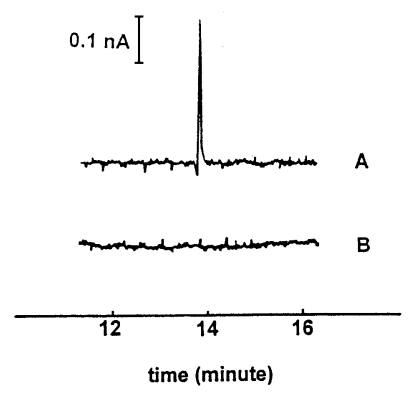

Fig. 4 Electropherograms of $5.0 \mu \mathrm{M}$ CYS obtained at CoP/PME (A) and blank PME (B). Conditions as in Fig. 3.

detection potential was set at $1.0 \mathrm{~V}$ for the PME and $1.2 \mathrm{~V}$ for the platinum microelectrode and CFME. As shown in Fig. 2, no electrophoretic peak was obtained for CYS and GSH at the platinum microelectrode and CFME. However, well-defined electrophoretic peaks were observed for CYS and GSH at the CoP/PME. The signal enhancement at PME compared to CFME and platinum microelectrode results from two aspects. On the one hand, PME has a porous matrix. It offers higher specific surface area and more effective binding site for analytes than the other two microelectrodes, so the oxidation currents for analytes were enhanced. On the other hand, the electrocatalytic activity of CoP packed in PME might contribute to the increase in current response (to be discussed later). Hence, the detection sensitivity of thiols is improved by using CoP/PME instead of CFME or platinum microdisk electrodes.

Hydrodynamic voltammograms were used to investigate the effect of electrode potential on the detection sensitivity. Shown in Fig. 3 is the hydrodynamic voltammogram of CYS. It can be seen that the peak current for CYS increases as the detection potential is increased from $0.5 \mathrm{~V}$ to $1.0 \mathrm{~V}$. However, a plateau of the peak current was observed when the detection potential was $\geq 0.9 \mathrm{~V}$. So the optimum detection potential can be chosen as $1.0 \mathrm{~V}$.

We also compared the results obtained for CYS at CoP/PME and blank PME (only graphite powder packed). Figure 4 presents the electrophoregrams of $5.0 \mu \mathrm{M}$ CYS obtained at the CoP/PME (A) and blank PME (B). As shown in Fig. 4, the detection sensitivity was improved greatly at CoP/PME since cobalt porphyrin packed in PME has the electocatalytic activity toward CYS. In comparison with the electrophoretic peak of $500 \mu \mathrm{M}$ CYS shown in Fig. 2, it can be observed that the peak
Table 1 Statistical data of CYS detection by using CEamperometry with CoP/PME

\begin{tabular}{ccccc}
\hline$N$ & CLOD/nM & MLOD/amol & RSD $_{\mathrm{p}}, \%$ & $\mathrm{RSD}_{\mathrm{m}}, \%$ \\
\hline 150000 & 88 & 230 & 1.8 & 1.0 \\
\hline
\end{tabular}

$N$ : theoretical plate number; CLOD: concentration limit of detection; MLOD: mass limit of detection; $\mathrm{RSD}_{\mathrm{p}}$ : relative standard deviation of peak heights $(n=8) ; \operatorname{RSD}_{\mathrm{m}}$ : relative standard deviation of migration time $(n=8)$.

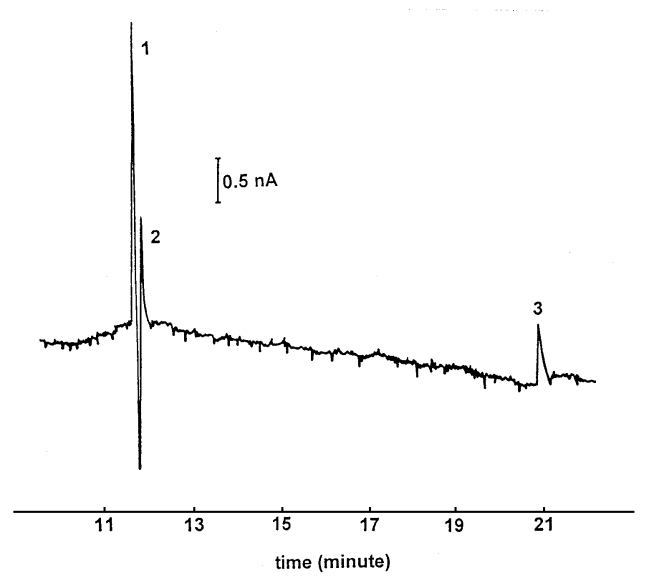

Fig. 5 Electropherogram of $100 \mu \mathrm{M}$ hydrazine (1), hydroxylamine (2), and 2-mercapto ethanol (3) obtained at CoP/PME. Buffer, 20 $\mathrm{mM}$ phosphate ( $\mathrm{pH} 6.8)$; injection, $10 \mathrm{~s}$ at $10 \mathrm{~cm}$. Other conditions as in Fig. 3.

current of CYS is only one order of magnitude less, while its concentration decreases by two orders of magnitude. Table 1 lists the statistical data of CYS by using CE-amperometry with $\mathrm{CoP} / \mathrm{PME}$. It should be pointed out that CoP/PME provides very reproducible responses to CYS. Passivation of the electrode often occurs since the oxidation products of analytes often adsorb strongly on the electrode surface (e.g. thiols). Pulse amperometric detection can overcome this problem by combining amperometric detection with attenuated anodic and cathodic polarizations to clean and activate the electrode surface, but this technique is usually applied to activate $\mathrm{Au}$ or $\mathrm{Pt}$ microelectrodes (most for carbohydrates or alcohols). In our experiments, severe fouling of CFME and platinum microelectrodes caused by the electro-oxidation of CYS and GSH was observed. Compared to the first injection, the response for the second injection reduced dramatically because the oxidation products of these two thiols were adsorbed strongly on the electrode surface. Therefore, it is impossible to get reproducible peaks for CYS and GSH at CFME and platinum microelectrodes, even though the concentration of analytes is increased to a high level. In contrast, thiols show reproducible electrophoretic peaks at the CoP/PME, indicating that the electrode fouling of thiols can be effectively reduced by packing PME with cobalt porphyrin. Since the mediated electro-oxidation of thiols by cobalt porphyrin complex might change the adsorption characteristics of the oxidation products dramatically, the CoP/PME shows high resistance to poisoning.

The CoP/PME was also applied to detect hydrazine, hydroxylamine and 2-mercaptoethanol. Shown in Fig. 5 is the electrophoregram of these three analytes obtained in phosphate buffer ( $\mathrm{pH}$ 6.8). The corresponding plate numbers are determined to be 110000,230000 , and 180000 , respectively. If 
one compares with the electrophoregram obtained in MES buffer, the detection noise in phosphate buffer increases since the current in the separation capillary increases. Detection limits of hydrazine, hydroxylamine and 2-mercaptoethanol were calculated to be $1.4 \times 10^{-6}, 4.0 \times 10^{-6}$, and $6.6 \times 10^{-6}$, respectively $(S / N=3)$.

We have demonstrated the application of PME in CEamperometry and have outlined its major advantages of high detection sensitivity, good reproducibility and renewability. This type of microelectrode has been used for the detection of thiols, hydrazine and hydroxylamine, and we are confident that it will be applicable to many other compounds of biological interest.

\section{References}

1. A. G. Ewing, J. M. Mesaros, and P. F. Gavin, Anal. Chem., 1994, 66, 527A.

2. Y. F. Yik and S. F. Y. Li, Tr. Anal. Chem., 1992, 11, 325.

3. A. G. Ewing, J. Neurosci. Methods, 1993, 48, 215.

4. W. Lu and R. M. Cassidy, Anal. Chem., 1993, 65, 2878.
5. J. Wen and R. M. Cassidy, Anal. Chem., 1996, 68, 1047.

6. J. Ye and R. P. Baldwin, Anal. Chem., 1994, 66, 2669.

7. C. E. Engstrom-Silverman and A. G. Ewing, J. Microcol. Sep., 1991, 3, 141.

8. W. Lu and R. M. Cassidy, Anal. Chem., 1993, 65, 1649.

9. T. J. O'Shea and S. M. Lunte, Anal. Chem., 1993, 65, 247.

10. X. Huang and W. T. Kok, J. Chromatogr., 1995, 716, 347.

11. J. Liu, W. Zhou, T. You, F. Li, and E. Wang, Anal. Chem., 1996, 68, 3350.

12. C. S. Cha, C. M. Li, H. X. Yang, and P. F. Liu, J. Electroanal. Chem., 1994, 368, 47.

13. P. F. Liu, C. S. Cha, X. Li, and J. Chen, Chem. J. Chin. Univ., 1994, 15, 725.

14. S. Hu, D. W. Pang, Y. Hu, and J. K. Cheng, Chem. Res. Chinese Univ., 1997, 13, 221.

15. S. Hu, Z. L. Wang, P. B. Li, and J. K. Cheng, Anal. Chem., 1997, 69, 264.

16. D. W. Pang and Z. L. Wang, J. Electroanal. Chem., 1993, 358, 235.

17. D. W. Pang, Z. L. Wang, and C. S. Cha, J. Electroanal. Chem., 1992, 325, 219.

18. Z. Y. Deng, Z. L. Wang, C. M. Li, and C. S. Cha, Chem. J. Chin. Univ., 1987, 8, 453. 